\title{
Prevention of Medication Errors in a Pakistani Hospital Because of Concurrent Evaluations and Interventions by Pharmacists
}

\author{
Hassan Raza ${ }^{1 *}$, Yasmin Akhtar ${ }^{2}$ \\ ${ }^{1}$ Bone Marrow Transplant Unit, Dow University of Health Sciences, Karachi, Pakistan. \\ ${ }^{2}$ Department of Pharmacy Services, The Indus Hospital, Karachi, Pakistan.
}

\begin{abstract}
Objective: The aim of this study is to document different pharmaceutical interventions that took place during dispensing through Computerized Physician Order Entry (CPOE) to prevent medication errors at a multidisciplinary tertiary care hospital in Karachi, Pakistan.
\end{abstract}

Method: Study Design: Systematic retrospective review of e-prescriptions received in inpatient pharmacy for dispensing in a multidisciplinary tertiary care hospital.

Dispensing Setting: In order to decrease medication administration error, the hospital works on Unit Dose Dispensing System, all the orders are segregated by Health Management Information Software and appeared on inpatient pharmacist window for dispensing, the inpatient pharmacist then check all the medication order, if the pharmacist find any medication error or better alternative, the pharmacist contact the concerned physician and discuss about the error and suggest alternatives, if the physician find the suggestion beneficial for the patient then the order is intervened.

Date Collection: These interventions were recorded by in-patient pharmacists from June 2014 till May 2015, which were then filled in a performa and categorized for potential of harm if not intervened.

Result: A total of 1336 interventions are accepted during the study period. About $83.2 \%$ of the interventions were found to be minor or moderately harmful. Most significant intervention was related to dose correction (26.9\%), followed by drug alternatives (therapeutic or brand alternative) (26.6\%), wrong frequency of drug (20.7\%), excessive duration (9.2\%), drug duplication (6.2\%), culture and sensitivity based drugs $(2.3 \%)$, wrong choice of drug $(1.9 \%)$, wrong route $(0.7 \%)$, wrong dosage form $(0.7 \%)$, Addition of drug $(0.2 \%)$ and drug-drug interactions $(0.1 \%)$.

Conclusion: This study shows that concurrent evaluation of prescription by pharmacist decreases preventable medication errors which probably decrease health care cost and decrease patient hospital stay.

Keywords: Physician order entry, Drug dispensing, Inpatient pharmacy, Patient oriented care, Pharmaceutical intervention.

\section{BACKGROUND}

The role of the pharmacist has been recognized all over the world in providing patient focused care [1]. Participation of pharmacist in clinical rounds, reviewing patients medical records, reconciliation of medication, and patient counselling about medications on discharge and follow up results in improve clinical outcomes [2], decrease mortality rates [3] and reduces preventable adverse drug reactions [4].

But in developing countries like Pakistan, the doctor of pharmacy program is more industry oriented than hospital or patient care oriented [5] and there are very few institutions who train pharmacist for clinical services that's why hospital or clinical pharmacist struggles to play their role in patient care. This is a reason why hospital or community pharmacy in

\footnotetext{
*Address correspondence to this author at the Bone Marrow Transplant Unit, Dow University of Health Sciences, Karachi, Pakistan.

E-mail: rph.hassan@hotmail.com
}

Pakistan is miserable with few qualified pharmacist in hospital and community pharmacy settings [6, 7]. Though pharmacy services are evolving but instead of current pharmacy evolution in Pakistan, pharmacist in hospital and community are more engaged with inventory management [8], with the exception of few hospitals where pharmacist is the integral part of patient focused care.

The knowledge of medication errors is essential prerequisite for better patient care, these medication error remains unnoticed in majority of health care centres of Pakistan due to manual or semi electronic prescribing system [9]. These errors can be minimize by pharmacy interventions that are thought to be the integral part of recent pharmacy services inspiring the standard of care and prevent major organ damage and potential life threatening conditions [10] and are known to decrease the patient medication cost contribute in rationalization of medication therapy [11], medicine 
adherence [12]. and significantly decreases the mortality rate [13] but there is very limited data available from Pakistan.

\section{OBJECTIVE}

This study aims to document different pharmaceutical interventions that took place during dispensing through Computerized Physician Order Entry (CPOE) to prevent medication error at a multidisciplinary tertiary care hospital in Karachi, Pakistan.

\section{METHODOLOGY}

\section{Study Design}

Systematic retrospective review of e-prescriptions received in inpatient pharmacy for dispensing.

\section{Study Setting \& Target Population}

The study is conducted in a multidisciplinary tertiary care facility situated in Korangi, having 150 beds reserved for inpatient settings, six beds are reserved for emergency and six for day care. The dispensing is done through computerized physician order entry with Institutional Health Management Information Software (HMIS).

\section{Study Period}

Though the concurrent evaluation of prescription is continuous process but the interventions which had done from June 2014 till May 2015 during unit dose dispensing by inpatient pharmacist, are included. The data of unit solar year is chosen so it covers all the seasons.

\section{Sample Size}

All prescriptions meeting inclusion and exclusion criteria.

\section{Sampling Selection}

\section{Inclusion Criteria}

Interventions done by inpatient pharmacist during dispensing through CPOE.

\section{Exclusion Criteria}

Interventions done by clinical pharmacist, antibiotic stewardship team, infection control team or intervention during dispensing in emergency pharmacy and OPD, or interventions done in other campuses of the hospital were excluded.

\section{Data Collection Procedure}

In order to decrease medication administration error, the hospital works on unit dose dispensing system, all the orders are segregated by HMIS and appeared on trained inpatient pharmacist window for dispensing. The inpatient pharmacist then check all the medication order, if the pharmacist find any medication error or better alternative, the pharmacist contact the concerned physician and discuss about the error and suggest alternatives. If the physician find the suggestion beneficial for the patient then the order is intervened.

During the study period, all inpatient pharmacists were instructed to save pharmacy interventions. Data of All interventions were then filled in performa manually by pharmacist and trainee pharmacist. These interventions were then sorted by senior clinical pharmacist for potential of harm that can be caused if not intervened.

a. Minor will be those that do not harm the patient and need monitoring.

b. Moderate will be those that can cause a temporary harm if used.

c. Major will be those that can result in permanent harm to any organ, near-death or death.

\section{Ethical Consideration}

All ethical aspects concerning the study were observed.

\section{Statistical Analysis}

The data is first entered in MS excel then in SPSS ver. 20 for statistical analysis.

\section{RESULT}

\section{Demography}

More number of interventions are concerned with adults (21 years or above) about $65.3 \%$ followed by children ( 2 months - 12 year) $18 \%$ and adolescent (13 year - 20 Year) 9.1\%. interventions related to infants (7.6\%) and new-borns $(0.2 \%)$ were very minimum. $60 \%$ of total Interventions were done on prescriptions of male patients while $40 \%$ were female. The paediatric age group is referred from Pediatric Expertise for Advisory Panels; Guidance for Industry and FDA Staff [14].

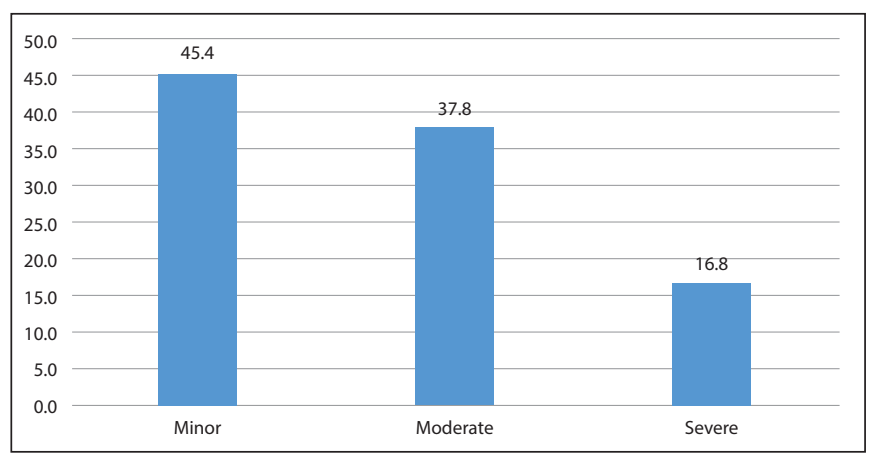

Fig. (1). Potential Harm of Medication Error.

\section{TYPES OF MEDICATION ERROR AND THEIR SEVERITY}

A total of 1336 Interventions were accepted during the study period. But only $16.8 \%$ was found to be sever if not intervened and $83.2 \%$ were found to be minor or moderately 
Table 1. Types of Medication Error and their Potential Harm.

\begin{tabular}{|l|c|c|c|c|}
\hline & Minor & Moderate & Severe & Total \\
\hline Non-Renal Adjusted Dose & 2 & 24 & 32 & 58 \\
\hline Wrong Dosage Form & 8 & 1 & 1 & 10 \\
\hline Excessive Duration of then Required & 17 & 70 & 36 & 123 \\
\hline Wrong Drug & 7 & 15 & 4 & 26 \\
\hline Wrong Route & 7 & 1 & 1 & 9 \\
\hline Drug Alternative / Substitute & 356 & 0 & 0 & 356 \\
\hline Drug Duplication & 8 & 55 & 20 & 83 \\
\hline Addition Of Drug & 2 & 1 & 0 & 3 \\
\hline Culture and Sensitivity Based Drug & 1 & 9 & 21 & 31 \\
\hline Wrong Dose & 95 & 190 & 74 & 359 \\
\hline Wrong Frequency & 102 & 139 & 35 & 276 \\
\hline Drug-Drug Interaction & 2 & 0 & 0 & 2 \\
\hline Total & 607 & 505 & 224 & 1336 \\
\hline
\end{tabular}

harmful as shown in Fig. (1). The major area of intervention related to dose correction were about $26.9 \%$ (359), and found to be moderately harmful if not intervened. Switching to alternative drug (pharmaceutical alternative $4.7 \%$, therapeutic alternative $20.3 \%$ ) to adhere the physicians to hospital drug formulary, and to reduce cost was found to be $26.6 \%$ (356), followed by wrong frequency (20.7\%). Excessive duration $(9.2 \%)$, drug duplication $(6.2 \%)$, renal adjusted dose $(4.3 \%)$, culture and sensitivity based drug $(2.3 \%)$, wrong drug entered in notes (1.9), wrong dosage form $(0.7 \%)$, wrong route of administration $(0.3 \%)$ addition of drug $(0.2 \%)$ and drug-drug interaction $(0.1 \%)$ as shown in (Table $\mathbf{1})$.

\section{TYPES OF PHARMACEUTICAL INTERVENTION}

All interventions are divided into four categories as shown in Table 2. A total of $568(42.5 \%)$ interventions were related to change in drug or the dosage form of the drug. While 399 $(29.9 \%)$ interventions brought change in dose either by increasing due to sub-therapeutic dose $(11.4 \%$ of total) or decreasing $(18.7 \%)$ due to renal adjustment or supra-therapeutic dose, followed by wrong frequency (282) $(21.1 \%)$ and drug stop due to duration or any other reason (87) $(6.5 \%)$.
Table 2. Types of Pharmaceutical Intervention.

\begin{tabular}{|l|c|c|c|c|}
\hline & Frequency & Percent & $\begin{array}{c}\text { Valid } \\
\text { Percent }\end{array}$ & $\begin{array}{c}\text { Cumulative } \\
\text { Percent }\end{array}$ \\
\hline $\begin{array}{l}\text { Drug Stop Due } \\
\text { to any Reason }\end{array}$ & 87 & 6.5 & 6.5 & 6.5 \\
\hline $\begin{array}{l}\text { Change in Dosing } \\
\text { Frequency }\end{array}$ & 282 & 21.1 & 21.1 & 27.6 \\
\hline Change in Dose & 399 & 29.9 & 29.9 & 57.5 \\
\hline $\begin{array}{l}\text { Change or Addition } \\
\text { of Drug }\end{array}$ & 568 & 42.5 & 42.5 & 100.0 \\
\hline Total & 1336 & 100.0 & 100.0 & \\
\hline
\end{tabular}

\section{DISCUSSION}

This study shows that pharmacy intervention prevent drug related harm. Though most of the interventions are found to be minor or moderately harmful if not intervened but it surely save cost of therapy and enhance quality of care as shown in studies $[15,16]$. This study focuses the role of inpatient dispensing pharmacist in Pakistan though there are number of studies published which elaborate the role of clinical pharmacist $[17,18]$ and community pharmacist $[19,20]$. The high rate of accepted pharmacy intervention (111.3 interventions / month) shows the role of inpatient dispensing 
pharmacist in providing quality and patient focused care. Most of the intervention are related to adult patients (65.3\%) because less numbers of beds are reserved for paediatric patients. Since the hospital work with computerized physician order entry CPOE and computerized dispensing so, it is expected to have less medication error and more interventions [21]. The census 2017 shows that pakistan has more males then female, that could be a reason for male dominance in this study as well [22].

Most of the interventions presented minor $(45.4 \%)$ or moderate harm (37.8) while only $16.8 \%$ was found to be severe if not intervened which is just similar to the study done by HAM Al Rhab et al. [23] The major interventions are concerned with inappropriate dose which supports the study done in Austrailian teaching hospital having $43.6 \%$ of interventions concerned with high doses [24]. The importance of this study can be realize by the data obtained having $70.8 \%$ interventions prevent toxicity $(35.3 \%)$ and rationalize the treatment $(35.5 \%)$.

We recommend future researches on the cost saved by inpatient pharmacist during unit dose dispensing through $\mathrm{CPOE}$, we also recommend further research on different factor which contribute in the success of any pharmacy intervention. Though this study shows the role of pharmacist in providing quality care, still we feel that pharmacist role in Pakistan is very limited. Therefore, we recommend a proper training or residency program for pharmacist.

\section{LIMITATIONS}

We hadn't recorded the interventions which were rejected by Primary Physician that's why we were unable to calculate the acceptance rate as done by Barber et al. [25]. Similarly we haven't analysed the no. of minutes spent on these Intervention as calculated by Hawkey et al. [26].

\section{CONCLUSION}

This study shows that concurrent evaluation of prescription by pharmacist decrease preventable medication errors. A total of 1336 interventions shows the crucial role of pharmacist in providing quality care but a proper national guidelines and training for inpatient and clinical pharmacist can improve the health care standard all over Pakistan. Because these interventions and pharmacy evaluation of prescriptions play a crucial role to prevent drug related harm. Continuous pharmacist review also improves the hospital prescribing practise because inpatient pharmacist is in position to educate health care practitioners about drugs.

\section{CONTRIBUTION OF AUTHORS}

Hassan Raza and Yasmin Akhtar conceived and designed the study. Both the authors have equally contributed in collection and analysis of data. Hassan Raza has wrote the paper while Yasmin Akhtar has reviewed it and have made changes. authorship must be limited to both.

\section{CONFLICT OF INTEREST}

Declared none.

\section{ACKNOWLEDGEMENTS}

We take this opportunity to thank pharmacy manager Dr. Aslam Shah and assistant pharmacy manager Dr. Zareena Khalid to provide all required facilities. To, our inpatient pharmacists Dr. Suleman, Dr. Ahmad, Dr. Ayaz, Dr. Kiran Jawed, Dr. Nosheen, Dr. Eraj Arfeen, Dr. Imrana Naqvi and Dr. Salma, for recording pharmacy intervention data. Also, our pharmacy trainees Dr. Midhat Jafri, Dr. Madiha Sana Fatima, Dr. Hiba, Dr. Tooba for helping us in compiling of data and everybody who gave us time and skills for this article.

\section{REFERENCES}

[1] Halvorsen KH, Stensland P, Granas AG. A qualitative study of physicians' and nurses' experiences of multidisciplinary collaboration with pharmacists participating at case conferences. Int J Pharm Pract 2011; 19(5): 350-7. DOI:10.1111/j.2042-7174.2011.00129.x

[2] Kaboli PJ, Hoth AB, McClimon BJ, Schnipper JL. Clinical pharmacists and inpatient medical care: A systematic review. Arch Int Med 2006; 166(9): 955-64. DOI:10.1001/archinte.166.9.955

[3] Bond C, Raehl CL. Clinical pharmacy services, pharmacy staffing, and hospital mortality rates. Pharmacother: J Hum Pharm Drug Therap 2007; 27(4): 481-93. DOI:10.1592/phco.27.4.481

[4] Kucukarslan SN, Peters M, Mlynarek M, Nafziger DA. Pharmacists on rounding teams reduce preventable adverse drug events in hospital general medicine units. Arch Int Med 2003; 163(17): 2014-8. DOI:10.1001/archinte.163.17.2014

[5] Bhagavathula AS, Sarkar BR, Patel I. Clinical pharmacy practice in developing countries: Focus on India and Pakistan. Arch Pharm Pract 2014; 5(2): 91. DOI:10.4103/2045-080X.132661

[6] Butt ZA, Gilani AH, Nanan D, Sheikh AL, White F. Quality of pharmacies in Pakistan: A cross-sectional survey. Int J Qual Health Care 2005; 17(4): 307-13. DOI:10.1093/intqhe/mzi049

[7] Rabbani F, Cheema F, Talati N, et al. Behind the counter: Pharmacies and dispensing patterns of pharmacy attendants in Karachi. JPMA: J Pakistan Med Assoc 2001; 51(4):149.

[8] Azhar S, Hassali MA, Ibrahim MIM, Ahmad M, Masood I, Shafie AA. The role of pharmacists in developing countries: The current scenario in Pakistan. Hum Res Health 2009; 7(1): 54. DOI:10.1186/1478-4491-7-54

[9] Riaz MK, Hashmi FK, Bukhari NI, Riaz M, Hussain K. 
Occurrence of medication errors and comparison of manual and computerized prescription systems in public sector hospitals in Lahore, Pakistan. PloS One 2014; 9(8): e106080. DOI:10.1371/journal.pone.0106080

[10] Chin J, Muller R, Lucarelli C. A pharmacy intervention program: recognizing pharmacy's contribution to improving patient care. Hosp Pharm 1995; 30(2): 120, 123-6, 129-30.

[11] Khalili H, Karimzadeh I, Mirzabeigi P, Dashti-Khavidaki S. Evaluation of clinical pharmacist's interventions in an infectious diseases ward and impact on patient's direct medication cost. Eur J Int Med 2013; 24(3): 227-33. DOI:10.1016/j.ejim.2012.11.014

[12] Murray MD, Young J, Hoke S, et al. Pharmacist intervention to improve medication adherence in heart failure A randomized trial medication adherence in heart failure. Ann Int Med 2007; 146(10): 714-25.

DOI:10.7326/0003-4819-146-10-200705150-00005

[13] Pottegård A, Hallas J, Søndergaard J. Pharmaceutical interventions on prescription problems in a Danish pharmacy setting. Int J Clin Pharm 2011; 33(6): 1019-27. DOI: $10.1007 / \mathrm{s} 11096-011-9580-4$

[14] Pediatric Expertise for Advisory Panels. Health CFD, Radiological. Search for FDA Guidance Documents Guidance for Industry and FDA Staff 2017.

[15] Cowper PA, Weinberger M, Hanlon JT, et al. The cost-effectiveness of a clinical pharmacist intervention among elderly outpatients. Pharmacother: J Hum Pharm Drug Therap 1998; 18(2): 327-32.

[16] Kopp BJ, Mrsan M, Erstad BL, Duby JJ. Cost implications of and potential adverse events prevented by interventions of a critical care pharmacist. Am J Health Syst Pharm 2007; 64(23): 2483-7. DOI:10.2146/ajhp060674

[17] Razi ZST, Latif SA, Shamim RS. Establishing clinical pharmacy services in a Pakistani intensive care unit. Am J
Health Syst Pharm 2002; 59(19): 1888-9.

[18] Jameson J, VanNoord G, Vanderwoud K. The impact of a pharmacotherapy consultation on the cost and outcome of medical therapy. J Fam Pract 1995; 41(5): 469-73.

[19] Ali I, Khan TM. Potential for community pharmacies to promote rational drug use in Pakistan. Res Soc Admin Pharm 2016; 12(1): 167-8. DOI:10.1016/j.sapharm.2015.09.003

[20] Khan SA, Motala MI, Khan MI, Sahad S. Time for pharmacists to accept their role in community pharmacy: Pakistani perspective. Res Soc Admin Pharm 2016; 12(2): 361-3. DOI:10.1016/j.sapharm.2015.10.006

[21] Radley DC, Wasserman MR, Olsho LE, Shoemaker SJ, Spranca MD, Bradshaw B. Reduction in medication errors in hospitals due to adoption of computerized provider order entry systems. J Am Med Inform Assoc 2013; 20(3): 470-6. DOI:10.1136/amiajnl-2012-001241

[22] Pakistan Bureau of Statistics. Provisional Summary Results Of 6th Population And Housing Census-2017. 6th Population and Housing Census 2017.

[23] Al Rahbi HAM, Al-Sabri RM, Chitme HR. Interventions by pharmacists in out-patient pharmaceutical care. Saudi Pharm J 2014; 22(2): 101-6. DOI:10.1016/j.jsps.2013.04.001

[24] Alderman CP, Farmer C. A brief analysis of clinical pharmacy interventions undertaken in an Australian teaching hospital. J Qual Clin Pract 2001; 21(4): 99-103. DOI:10.1046/j.1440-1762.2001.00428.

[25] Barber ND, Batty R, Ridout DA. Predicting the rate of physician-accepted interventions by hospital pharmacists in the United Kingdom. Am J Health Syst Pharm 1997; 54(4): 397-405.

[26] Hawkey C, Hodgson S, Norman A, Daneshmend T, Garner S. Effect of reactive pharmacy intervention on quality of hospital prescribing. BMJ 1990; 300(6730): 986-90. DOI:10.1136/bmj.300.6730.986 